\section{Updates from the Neuro-Oncology Section of the 2015 American Neurological Association Annual Meeting}

\author{
Rimas V Lukas ${ }^{*, 1}$, Derek A Wainwright ${ }^{2,3}$ \& John J Laterra ${ }^{4,5}$
}

\author{
American Neurological Association Annual Meeting, Chicago, IL, USA, 27-29 September \\ 2015
}

The American Neurological Association (ANA) held its annual meeting in Chicago, IL, USA on 27-29 September 2015. The Scientific Programming Advisory Committee was chaired by Dr S Pleasure from the University of California-San Francisco (CA, USA). The Neuro-Oncology session, chaired by Dr A Pruitt from the University of Pennsylvania (PA, USA) and cochaired by Dr J Laterra from Johns Hopkins University (MD, USA), was held on 27 September 2015. Speakers included Dr D Wainwright (Northwestern University, IL, USA), Dr N Kolb (University of Utah, UT, USA), Dr A Nath (NINDS/NIH, MD, USA), Dr D Franz (Cincinnati Children's Hospital, $\mathrm{OH}, \mathrm{USA}$ ) and Dr R Lukas (University of Chicago, IL, USA). A summary of key presentations from the Neuro-Oncology section of the 2015 American Neurological Association annual meeting is reported. Preclinical and clinical advances in the use of immunotherapies for the treatment of primary and metastatic CNS tumors are covered. Particular attention is paid to the enzyme indoleamine dioxygenase and the immune checkpoints CTLA4 and PD1 and their ligands. Specific nervous system toxicities associated with novel immunotherapies are also discussed. The recent success of targeting the mTOR pathway in the neurocutaneous syndrome tuberous sclerosis is detailed. Finally, important early steps in our understanding of the common toxicity of chemotherapy induced neuropathy are reviewed.

First draft submitted: 21 October 2015; Accepted for publication: 22 October 2015; Published online: 30 November 2015

Immunology in neuro-oncology

Glioblastoma (GBM) is a highly immunosuppressive primary brain tumor of the CNS. Among the pathways that contribute to immune evasion, indoleamine 2,3 dioxygenase 1 (IDO), a rate-limiting enzyme that catabolizes tryptophan (Trp) into kynurenines (Kyn), is expressed in a majority of human GBM, in situ [1,2], as well as in GBM mouse models, where it has been demonstrated to mediate the accumulation of immunosuppressive Tregs $\left(\mathrm{CD} 4{ }^{+} \mathrm{CD} 25^{+} \mathrm{FoxP} 3^{+}\right)$[3]. This immunosuppressive microenvironment is further supported by the expression of the PD1 ligand, PDL1 (B7H1) on GBM cells [4] and tumor-infiltrating macrophages [5]. The binding of PD1 on T cells to PDL1 or PDL2 (B7DC) leads to either anergy or T-cell death. A complete understanding of PDL1/PDL2,

'Department of Neurology, University of Chicago, Chicago, IL, USA

2Department of Neurological Surgery, Northwestern University, IL, USA

${ }^{3}$ Northwestern Brain Tumor Institute, Chicago, IL, USA

${ }^{4}$ Department of Neurology, Johns Hopkins University, Baltimore, MD, USA

${ }^{5}$ Sidney Kimmel Comprehensive Cancer Center, Baltimore, MD, USA

*Author for correspondence: Tel.: +1 773834 9026; Fax: +1 773702 7485; rlukas@neurology.bsd.uchicago.edu 
including possible T-cell activation facilitated by their binding to an as yet unspecified ligand, has yet to be elucidated. PDL1 has been shown to be expressed by the majority of newly diagnosed and recurrent GBMs, but not in the adjacent brain parenchyma. Its expression does not appear to be a prognostic factor for survival [6]. Due to clinical efficacy of CTLA4 [7] and PD1 [8] blockade in other tumors, and the substantial role of these receptors in immunosuppression associated with CNS tumors, there has been significant interest in evaluating these agents in high-grade gliomas and CNS metastases. The utilization of the CTLA4 antibody, ipilimumab in melanoma patients with brain metastases has demonstrated similar CNS and extra-CNS response rates $(24 \%)$ in patients with no prior CNS-directed therapy, but less robust response rates $(10 \%)$ in those with symptomatic brain metastases requiring steroids [7]. Of additional interest is potential synergy with other treatments such as radiotherapy in this patient population. An example of this is the abscopal effect, associated with improved survival, in patients with brain metastases treated with ipilimumab who subsequently received radiotherapy to the brain for progression of disease there. Some of these patients were observed to develop subsequent regression of systemic tumors that did not receive radiation pointing toward an immunemediated benefit [9]. While there have been no trials in primary brain tumors reporting response rates following treatment with IDO, CTLA4, PD1 and/or PDL1-targeted therapies, numerous ongoing trials are evaluating these potential targets, with the majority utilizing antibodies in patients with either high-grade glioma or brain metastases [10]. Results of these studies are eagerly awaited.

As development of these novel therapies move forward, a number of potential limitations will need to be considered. The first is the interpretation of radiographic endpoints. With therapeutic efficacy intended to induce a robust immune response against tumor, there is a concern for potential pseudoprogression prior to radiographic response. An understanding of the incidence of this effect in association with various immunotherapies is currently unknown. The neuro-oncology community, however, acknowledges that a unique set of criteria will be needed in evaluating responses to these therapies [11]. This has prompted the ongoing development of criteria aimed at a composite immunotherapeutic response assessment in neuro-oncology. There is also a need for reliable biomarkers to predict responsiveness to therapy. While somewhat inconsistent between studies, in extra-CNS cancer, there is evidence of a correlation between tumor expression of PDL1 and responsiveness to PD1 blockade. However, whether this holds true for CNS tumors will require evaluation in prospective clinical trials. Other potential biomarkers under investigation include the presence of tumor-infiltrating lymphocytes (TILs), the circulating Kyn/Trp level [12], as well as composite biomarker profiles. An additional challenge to immunotherapy against malignancies in the CNS is the need for neuro-oncologists to become familiar and comfortable with a constellation of side effects/toxicities associated with immunemediated impact; which markedly differ from those commonly seen with cytotoxic and antiangiogenic therapies. With immune checkpoint inhibitors (ICI), common symptoms include general malaise, fatigue and a flu-like syndrome. Less frequent, but more concerning toxicities include autoimmunity which can lead to hypophysitis, SIADH, thyroiditis, uveitis, pneumonitis, colitis, as well as myositis and neuromuscular junction dysfunction. Even with more targeted immunotherapies such as engineered $\mathrm{T}$ cells, both transient and progressive neurologic dysfunction have been described. Dr Nath presented on both B- and T-cell-mediated CNS syndromes associated with novel immunotherapies. The first was a description of a patient cohort with extra-CNS cancer treated with $\mathrm{T}$ cells modified to express anti-CD19 chimeric antigen receptor (CAR) that developed a syndrome of transient expressive aphasia, myoclonus, frontal release signs and cogwheel rigidity. This occurred without an associated alteration of consciousness. All patients recovered completely [13]. Utilizing the Allen Brain Atlas [14] it was noted that focal CD19 expression is present in Broca's area. Coincidently, CD19 expression was also observed in cultured neurons. It was speculated that transient neurological dysfunction, with no permanent destruction of the affected neuroanatomical region, was mediated by Fab fragments released by proteolytic digestion of the CD19 CAR construct or by a substance secreted by the CAR T cells. The second marked CNS toxicity related to a novel immunotherapy mediated by $\mathrm{T}$ cells. Four of nine patients treated with $\mathrm{T}$ cells bearing MAGE-A3-restricted TCR developed post-treatment leukoencephalopathy. Two 
of the patients died. MRI evaluation revealed diffuse areas of increased signal on FLAIR in the subcortical white matter. CSF analyses demonstrated the presence of modified $\mathrm{T}$ cells bearing the MAGE-A3 TCR, indicating trafficking of the therapeutic $\mathrm{T}$ cells into the CNS. Autopsy revealed areas of focal leukoencephalopathy and spongiform changes with the presence of a limited amount of $\mathrm{CD} 8^{+} \mathrm{T}$ cells and histiocytes perivascularly. While the pathologic changes were pronounced, signs of inflammation were less profound. Molecular assays revealed the presence of a multiple MAGE-A family members, including MAGE-A3, within the brain that may have contributed toward the neuron-directed immune response [15].

The final issue which is actively being addressed is the optimization of immunotherapies. Preclinical work in experimental brain tumor models support a substantial benefit when therapies are administered combinatorially [16]. Further increased effectiveness may benefit by combining multiple ICI with active vaccination. Combination with traditional chemotherapies is also worth exploring with regard to timing and acceptable toxicity. While studies combining vaccine therapies with temozolomide have shown that immunotherapy can be timed in relation to depletion of Tregs by the cytotoxic chemotherapy, preclinical work has shown diminished effectiveness of temozolomide when added to combinatorial checkpoint blockade [16,17]. There is also a concern for a marked increase in serious toxicities when combining ICI with traditional cytotoxics, as highlighted by grade 3/4 levels (49\% of patients) when doublet chemotherapy was used concomitantly with PDL1 blockade [18] compared with rates of approximately $10 \%$ when PDL1 mAb was used as a single agent. As previously mentioned, the combination of ICI with radiotherapy holds promise for synergy and is currently being evaluated in clinical trials for brain metastases as well as newly diagnosed high-grade gliomas [10]. Combination with the antiangiogenic agent bevacizumab, which has steroid sparing effects and is commonly used in recurrent GBM, is also under investigation. Finally, the optimal sequencing of these treatment modalities will require further clarification.

\section{Targeted therapy in tuberous sclerosis}

Tuberous sclerosis (TS) is a neurocutaneous disorder driven by mTOR over activity due to mutation of TSC1/TSC2. It manifests clinically with hamartomas of numerous organ systems including the skin, kidneys, eyes, heart, lungs and brain. Approximately $20 \%$ of patients with TS develop subependymal giant cell astrocytomas (SEGA). These WHO grade I tumors can grow quite large and cause significant morbidity. Recent clinical advances have come to fruition with the US FDA approval of everolimus for the treatment of SEGAs which cannot be curatively resected in patients with TS. This approval was secondary to the results of the randomized Phase III EXIST1 trial, as well as other related studies presented by Dr Franz [1921]. EXIST1 enrolled 117 patients $(18 \%<3$ years old, $18 \% \geq 18$ years old) who were randomized to receive everolimus (5-15 $\mathrm{mg}$ daily) versus placebo. After the core phase of the trial, there was a crossover/open-label extension. Utilizing volumetric measurements, all patients receiving everolimus experienced some degree of response in the SEGAs. Approximately $2 / 3$ of patients qualified as partial or complete responses. A similar response rate was noted in the less explicitly quantified skin lesions. The majority of toxicities were low grade and there was a trend toward a decreased incidence of these as patients remained on drug for a longer time. There was no apparent delay in puberty in patients on study drug nor any apparent effect on somatic growth in the very young patients treated on study. Discussion entailed a number of concepts to reduce potential toxicities in this treatment for young patients with indolent but serious tumors. These strategies included the use of the lowest effective dose of drug and drug holidays/alternate dosing schedules.

\section{Chemotherapy-associated neuropathy}

Chemotherapy-induced peripheral neuropathy (CIPN) is an inadequately understood complication of many systemic chemotherapies including vinca alkaloids, taxanes and platinum agents used in the treatment of cancer. It often manifests as a length dependent sensorimotor neuropathy with frequent involvement of the upper extremities in addition to lower extremities. Autonomic involvement, with constipation as a frequent symptom, may also be present. CIPN can be a dose-limiting toxicity leading to chemotherapeutic dose reductions and/or delays. CIPN is associated with a substantial impairment in quality of life. Less clearly established is the risk factors for developing CIPN, how to most effectively prevent/treat CIPN, specific 
morbidity associated with CIPN and whether there is an increased mortality associated with it.

Interesting new work presented by Dr Kolb [22] addressed several of these questions, laying the groundwork for future studies. In one study, the ICD9 code data from a large number of patients with specific common cancer histologies was evaluated for the presence of a range of neuropathy codes prior to the initiation of chemotherapies and in the 2-year interval following their initiation. Two key results emerged: across various histologies, a number of patients were coded as having developed neuropathy after the initiation of chemotherapy and the incidence of neuropathy was substantially undercoded compared with what would be expected for the general population. This points to the limitations of retrospectively analyzing large datasets of billing/coding information to definitively answer these questions. A second study evaluated the rate of falls associated with CIPN. Participants engaged in a daily phone log rating the severity of their symptoms and the incidence of falls. This frequent sampling method allows for a more nuanced picture of sustained symptom severity as well as a high likelihood of capturing the incidence of falls. Incidence of falls correlated with severity of symptoms from CIPN with $13 \%$ of patients with mild symptoms and $40 \%$ with moderateto-severe symptoms experiencing falls over the course of the study (hazard ratio: 2.96; $\mathrm{p}<0.003$ ). While patients with mild symptoms had a wide distribution of body parts injured with falls, those with moderate/severe symptoms experienced a disproportionate percentage of head injuries.

\section{Conclusion}

Recent advances in Neuro-Oncology have the potential to influence clinical management in multiple domains of this continuously evolving subspecialty. A clearer understanding of the incidence of CIPN and its consequences will be a key step in the development of therapeutic and more importantly preventative strategies for this common treatment related toxicity. The groundwork which has been laid in the field of cancer immunology is now manifesting in exciting translational and clinical investigations in primary and metastatic CNS tumors. These include the targeting of the enzyme IDO and the immune checkpoints CTLA4 and PD1/PDL1 in both preclinical models and early phase clinical trials. As these and other immunotherapies are investigated we will need to be aware of a new constellation of potential toxicities, some of them quite severe. With time, it is possible that we may see clinical responses in patients with high-grade gliomas and CNS metastases, to the same degree we now see with mTOR inhibition in tuberous sclerosis.

Financial \& competing interests disclosure

The authors have no relevant affiliations or financial involvement with any organization or entity with a financial interest in or financial conflict with the subject matter or materials discussed in the manuscript. This includes employment, consultancies, honoraria, stock ownership or options, expert testimony, grants or patents received or pending, or royalties.

No writing assistance was utilized in the production of this manuscript.

\section{References}

1 Uyttenhove C, Pillote L, Theate I et al. Evidence for a tumoral immune resistance mechanism based on tryptophan degradation by indoleamine 2,3-dioxygenase. Nat. Med. 9(10), 1269-1274 (2003).

2 Mitsuka K, Kawatake T, Satoh E, Asahara T, Horikoshi T, Kinouchi H. Expression of indoleamine 2,3-dioxygenase and correlation with pathological malignancy in gliomas. Neurosurgery 72(6), 1031-1038; discussion 1038-1039 (2013).

3 Wainwright DA, Balyasnikova IV, Chang AL et al. IDO expression in brain tumors increases the recruitment of regulatory $\mathrm{T}$ cells and negatively impacts survival. Clin. Cancer Res. 18(22), 6110-6121 (2012).
4 Parsa AT, Waldron JS, Panner A et al. Loss of tumor suppressor PTEN function increases B7-H1 expression and immunoresistance in glioma. Nat. Med. 13(1), 84-88 (2007).

5 Bloch O, Crane CA, Kaur R, Safaee M, Rutkowski MJ, Parsa AT. Gliomas promote immunosuppression through induction of B7-H1 expression in tumor-associated macrophages. Clin. Cancer Res. 19(12), 3165-3175 (2013).

6 Berghoff AS, Kiesel B, Widhalm G et al. Programmed death ligand 1 expression and tumor-infiltrating lymphocytes in glioblastoma. Neuro Oncol. 17(8), 1064-1075 (2015).

7 Margolin K, Ernstoff MS, Hamid O et al. Ipilimumab in patients with melanoma and brain metastases: an open-label, Phase 2 trial. Lancet Oncol. 13(5), 459-465 (2012).

8 Brahmer J, Reckamp KL, Baas P et al. Nivolumab versus docetaxel in advanced squamous-cell non-small-cell lung cancer. N. Engl. J. Med. 373(2), 123-135 (2015).

9 Grimaldi AM, Simeone E, Giannarelli D et al. Abscopal effects of radiotherapy on advanced melanoma patients who progressed after ipilimumab immunotherapy. Oncoimmunology 3, e28780 (2014).

10 Binder DC, Davis AA, Wainwright DA. Immunotherapy for cancer in the central nervous system: current and future directions. Oncoimmunology doi:10.1080/216240 2X.2015.1082027 (2015) (Epub ahead of print). 
11 Reardon DA, Okada H. Re-defining response and treatment effects for neuro-oncology immunotherapy clinical trials. J. Neurooncol. 123(3), 339-346 (2015).

12 Zhai L, Dey M, Lauing KL et al. The kynurenine to tryptophan ratio as a prognostic tool for glioblastoma patients enrolling in immunotherapy. J. Clin. Neurosci. (2015) In Press.

13 Kochenderfer JN, Dudley ME, Kassim SH et al. Chemotherapy-refractory diffuse large B-cell lymphoma and indolent B-cell malignancies can be effectively treated with autologous $\mathrm{T}$ cells expressing an anti-CD19 chimeric antigen receptor. J. Clin. Oncol. 33(6), 540-549 (2015).

14 Allen Brain Atlas. www.brain-map.org

15 Morgan RA, Chinnasamy M, Abate-Daga D et al. Cancer regression and neurological toxicity following anti-MAGE-A3 TCR gene therapy. J. Immunother. 36(2), 133-151 (2013).

Wainwright DA, Chang AL, Dey M et al. Durable therapeutic efficacy utilizing combinatorial blockade against IDO, CTLA-4 and PD-L1 in mice with brain tumors. Clin. Cancer Res. 20(20), 5290-5301 (2014).

17 Sampson JH, Aldape KD, Archer GE et al. Greater chemotherapy-induced lymphopenia enhances tumor-specific immune responses that eliminate EGFRvIII-expressing tumor cells in patients with glioblastoma. Neuro Oncol. 13(3), 324-333 (2011).

18 Rizvi NA, Antonia SJ, Chow LQM et al. A Phase I study of nivolumab (anti-PD1; BMS-936558; ONO-4538) plus platinumbased doublet chemotherapy (PT-doublet) in chemotherapy-naïve non-small cell lung cancer (NSCLC) patients (pts). J. Clin. Oncol. 31(Suppl), Abstract 8072 (2013).
19 Franz DN, Agricola A, Mays M, et al. Everolimus for subependymal giant cell astrocytoma: 5-year final analysis. Ann. Neurol. doi:10.1002/ana.24523 (2015) (Epub ahead of print).

20 Franz DN, Belousova E, Sparagana $S$ et al. Everolimus for subependymal giant cell astrocytoma in patients with tuberous sclerosis complex: 2-year open-label extension of the randomised EXIST-1 study. Lancet Oncol. 15(13), 1513-1520 (2014).

21 Franz DN, Agricola K, Mays M et al. Everolimus for subependymal giant cell astrocytoma: 5-year final analysis. Ann. Neurol. doi:10.1002/ana.24523. (2015) (Epub ahead of print).

22 Kolb N, Singleton JR, Brown S, CurtinK, Smith AG, Prevalence of chemotherapy induced peripheral neuropathy [abstract]. Ann. Neurol. doi:10.1002/ana.24498 (2015) (Epub ahead of print). 